\title{
Finite Capacity Scheduling of Make-Pack Production: Case Study of Adhesive Factory
}

\author{
Theppakarn Chotpradit ${ }^{1}$ and Pisal Yenradee ${ }^{2}$ \\ ${ }^{1}$ Master of Engineering Student, Logistics and Supply Chain Systems Engineering Program, School of Manufacturing \\ Systems and Mechanical Engineering, Sirindhorn International Institute of Technology, Thammasat University, Pathum- \\ Thani, 12121, Thailand. Email: theppakarn.chotpradit@ studentmail.siit.tu.ac.th \\ ${ }^{2}$ Associate Professor, Logistics and Supply Chain Systems Engineering Program, School of Manufacturing Systems and \\ Mechanical Engineering, Sirindhorn International Institute of Technology, Thammasat University, Pathum-Thani, 12121, \\ Thailand.Email: pisal@siit.tu.ac.th (corresponding author).
}

\author{
Production Management \\ Received January 27, 2014; revision April 10, 2014; accepted April 13, 2014 \\ Available online May 22, 2014
}

\begin{abstract}
This paper developed a Finite Capacity Scheduling (FCS) system for make-pack production based on a real case of an adhesive factory. The FCS determines production quantity of each machine to conform with resource capacities and due date of customer orders while minimizes related total cost. The total cost includes total production, inventory, and cleaning cost. A Mixed Integer Linear Programming (MILP) model is formulated and solved by LINGO software. The computational time is very long since the model has a lot of integer variables. Thus, the model is solved for a reasonable time and the best but not optimal solution is reported with the lower bound. This paper tries fixed horizon and rolling horizon scheduling methods. The fixed horizon plans for an entire horizon of 30 days. The rolling horizon plans for a sub-horizon of 10, 15, and 17 days. An overlapping of sub-horizons is applied to reduce end-of-horizon effect. Three scenarios (high, normal, and low) of demands are considered. The fixed horizon method is applied first to all scenarios of demand. If the best solution is far away from the lower bound, the rolling horizon method is applied. The results indicated that the rolling horizon method may significantly reduce the total cost with the same computational time. Moreover, the rolling horizon method is more applicable for a dynamic situation where customers frequently change orders. The proposed MILP model can generate reasonable solutions and they are useful for scheduling decision of makepack production.
\end{abstract}

Keywords: Make-pack, FCS, finite capacity scheduling, MILP, rolling horizon, scheduling optimization.

\section{Introduction}

A make-pack production is often applied in various industries such as shampoo, liquid detergent, and beverage industries. This paper considers the make-pack production in an adhesive factory which has two stages, namely, making and packing stages, which are buffered by an intermediate stage that has limited capacity as shown in Fig. 1.

This paper develops a Mixed Integer Linear Programming (MILP) model to solve make-pack production scheduling problem in the adhesive factory considering finite capacity of all work centers. Since the model is complex, the computational time is long. Thus, the model is solved for a reasonable time and the best but not optimal solution is reported with the lower bound. However, the obtained solution may be far away from the lower bound dependent on the data sets. This paper proposes a method to improve the quality of solution under acceptable computational time using a rolling horizon scheduling method.
The rolling horizon scheduling method is performed by dividing the entire planning horizon to smaller parts. First, solve the scheduling problem for the first horizon. Second, solve the scheduling problem for the second horizon by allowing an overlap between the two horizons. The aim of overlapping is to reduce the end of horizon effect between each horizon.

This paper also presents two different types of rolling horizon scheduling methods that are Rolling Horizon with Fixed Overlapping (RHFO) and Rolling Horizon with Variable Overlapping (RHVO). The RHVO will be applied in case that the solution of RHFO is infeasible.

This paper has objectives as follows.

1. To develop a Finite Capacity Scheduling (FCS) system for make-pack production based on a real case of an adhesive factory.

2. To develop the rolling horizon scheduling methods to improve the solution quality of the make-pack production scheduling. 


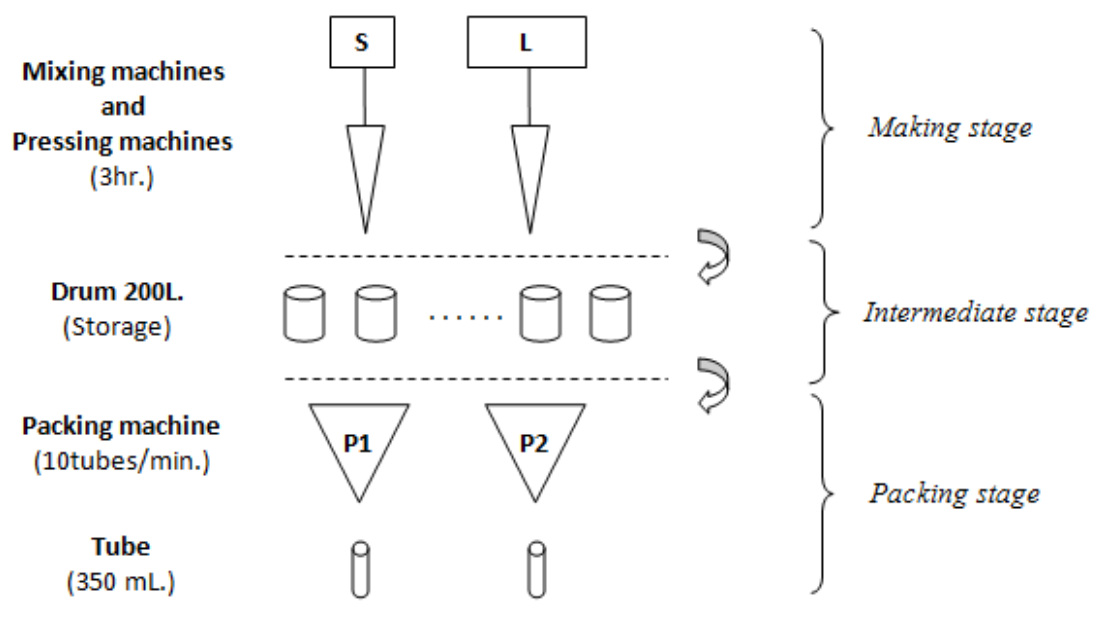

Fig. 1. Make-pack production system of adhesive

\section{Literature Reviews}

There are a number of research works that involve makepack problems such as Fundeling and Trautmann (2006), Gunther et al. (2006), and Mendez and Cerda (2002). Among these research works, an MILP approach is a widely used technique. Méndez and Cerdá (2002) developed an MILP continuous-time model for short-term scheduling to a make-pack continuous production plant by considering sequence-dependent setup times and due dates to meet all end-product demands at minimum make-span. Sun and Xue (2009) developed an MILP scheduling model based on a heuristic approach for the single-stage, multi-product batch plant with parallel units. The solution time of such scheduling model for the computational examples is much shorter than that of the existing models in their literatures when minimum makespan and total earliness of tasks are objective functions. Liu and Pinto (2010) used MILP based approaches for medium-term planning of single-stage continuous multiproduct plant with parallel units. Günther et al. (2006) applied two different approaches, namely, relaxed MILP model and Production Planning Detailed Scheduling using SAP APO software to solve make-pack production problem of hair dyes. The block planning concept and some alterative objective functions are also used in this paper. The makepack problem may be solved by heuristic methods. Wongthatsanekorn et al. (2013) applied bee colony optimization which is a meta-heuristic to solve make-pack production problem in process manufacturing of hair dye with an objective of minimizing the makespan. Honkomp et al. (2000) pointed out that the chemical process scheduling optimization problems in practice are difficult.

The make-pack scheduling is a kind of finite capacity scheduling (FCS) since it considers finite capacity of all work centers. Enns (1996) compared two different methods of FCS, blocked-time and event-drive, and conclude that event-drive is better than blocked-time method based on flow time and delivery performances.

The author also recommended about sending shop load information of FCS back to an MRP system to adjust planned lead times for more efficient planning. Nagendra and Das (2001) introduced FCS for solving MRP problem that considered capacity of available resources by specifying related constrains in MILP model together with specifying lot size for higher efficiency of MRP. Such approaches are recalled PCA (MRP progressive capacity analyzer).

When the scheduling problem is subject to uncertainties, a rolling horizon rescheduling strategy may be applied in dynamic environment. This strategy may be used to reduce computational time when planning for the entire horizon results in too long computational time. Fang and Xi (1997) adapted rolling horizon scheduling strategy to job shop production to solve dynamic environment problems where jobs arrive continuously, machines may breakdown, and due dates of jobs may change. Two problems in job shop scheduling, namely, dispatching operations to suitable machines and to deciding the processing sequence and release time of jobs on each machine, are separately solved with a hybrid scheduling algorithm that combines the genetic algorithm with the dispatching rules. Stauffer and Liebling (1997) applied rolling horizon scheduling algorithm based on tabu search in an aluminum manufacturing plant. The objective functions of scheduling are minimizing cumulative tardiness of all orders and maximizing rolling quality.

\section{Methodology and Model}

In this section, characteristics of the make-pack production process under consideration are briefly explained. Then, the MILP model is developed to determine optimal production plan and schedule. Finally, the rolling horizon planning methods are proposed to improve solution quality and reduce computational time.

\subsection{Production Process}

\section{Make stage}

- Ingredients are poured in a mixing machine

- Ingredients are mixed by mixing machine

- $\quad$ Mixed adhesive is pressed from mixing tank into drums by a pressing machine

- Close and seal each drum and wait for packing process

\section{Pack stage}

- $\quad$ Pack the mixed adhesive into small plastic tubes 


\subsection{MILP Model for Make-Pack Production Planning and Scheduling}

\subsubsection{Indices}

$p \quad$ index of product, $1,2, \ldots, P$; where $P$ is total number of products

$t \quad$ index of period, $1,2, . ., T$; where $T$ is planning horizon

$m \quad$ index of mixing machine, $1,2, . ., M$; where $M$ is total number of mixing machines

$n \quad$ index of packing machine, $1,2, \ldots, N$; where $N$ is total number of packing machines

\subsubsection{Parameters}

$\boldsymbol{B} \boldsymbol{S}_{\boldsymbol{p}, \boldsymbol{m}}$ batch size of product $p$ on mixing machine $m$ (units)

$\boldsymbol{C P}_{\boldsymbol{p}, \boldsymbol{m}}$ production cost of product $p$ on mixing machine $m$ (baht/batch)

$\boldsymbol{H} \boldsymbol{M}_{\boldsymbol{p}}$ inventory holding cost of intermediate product of product $p$ (baht/unit-period)

$\boldsymbol{H F}_{\boldsymbol{p}}$ inventory holding cost of finished goods of product $p$ (baht/unit-period)

$\boldsymbol{C C 1} \mathbf{1}_{\boldsymbol{p}}$ cleaning cost of product $p$ on mixing machines (baht)

$C C 2_{p}$ cleaning cost of product $p$ on packing machines (baht)

$\boldsymbol{D}_{\boldsymbol{p}, \boldsymbol{t}} \quad$ demand of product $p$ in period $t$ (units)

$\boldsymbol{I M}_{\boldsymbol{p}, \mathbf{0}}$ initial inventory of intermediate product of product $p$ (units)

$\boldsymbol{I M m a x}_{\boldsymbol{p}, \boldsymbol{t}}$ maximum inventory level of intermediate product of product $p$ in period $t$ (units)

$I_{p, 0} \quad$ initial inventory of product $p$ (units)

$\operatorname{Imin}_{\boldsymbol{p}, \boldsymbol{t}}$ minimum inventory level of product $p$ in period $t$ (units)

$\boldsymbol{B P}$ maximum number of mixing batches per period per machine (batches/period-machine)

$\boldsymbol{P} \boldsymbol{R}_{\boldsymbol{p}, \boldsymbol{n}}$ unit packing time of product $p$ on packing machine $n$ (minute/unit)

$\boldsymbol{A} \boldsymbol{P}_{\boldsymbol{n}}$ available packing time of packing machine $n$ (minute/period)

$\boldsymbol{S}_{\boldsymbol{n}} \quad$ set of products that can be packed on packing machine $n$

\subsubsection{Decision Variables}

$\boldsymbol{B}_{\boldsymbol{p}, \boldsymbol{m}, \boldsymbol{t}}$ number of batches of product $p$ on mixing machine $m$ in period $t$ (batches/period)

$\boldsymbol{I}_{\boldsymbol{p}, \boldsymbol{t}}$ ending inventory of intermediate product of product $p$ in period $t$ (units/period)

$\boldsymbol{I}_{\boldsymbol{p}, \boldsymbol{t}} \quad$ ending inventory of finished goods of product $p$ in period $t$ (units/period)

$\boldsymbol{P} \boldsymbol{Q}_{\boldsymbol{p}, \boldsymbol{n}, \boldsymbol{t}}$ packing quantity of product $p$ on packing machine $n$ in period $t$ (units/period)
First, indices, parameters, and variables of the model are defined. Then, the MILP model is formulated.

$\boldsymbol{M} \boldsymbol{M}_{\boldsymbol{p}, \boldsymbol{m}, \boldsymbol{t}} 1$, if product $p$ is produced on mixing machine $m$ in period $t$

0 , otherwise

$\boldsymbol{M} \boldsymbol{P}_{\boldsymbol{p}, \boldsymbol{n}, \boldsymbol{t}}$ 1, if product $p$ is packed on packing machine $n$ in period $t$

0 , otherwise

\subsubsection{Objective}

The objective of the model is to minimize total costs of production, cleaning mixing and packing machines, and inventory holding as shown in Eq. 1.

$$
\begin{aligned}
\min Z= & \sum_{p=1}^{P} \sum_{m=1}^{M} \sum_{t=1}^{T}\left(C P_{p, m} \cdot B_{p, m, t}+\right. \\
& \left.C C 1_{p} \cdot M M_{p, m, t}+C C 2_{p} \cdot M P_{p, n, t}\right)+ \\
& \sum_{p=1}^{P} \sum_{t=1}^{T}\left(H M_{p} \cdot I M_{p, t}+H F_{p} \cdot I_{p, t}\right)
\end{aligned}
$$

From Eq. 1, the first term is the summation of total production cost and total cleaning cost of mixing and packing machine, and the second term is summation of total inventory holding cost of intermediate and finished goods.

\subsubsection{Constraints}

Inventory balance:

$$
\begin{gathered}
I M_{p, t}=I M_{p, t-1}+\sum_{m=1}^{M} B_{p, m, t} \cdot B S_{p, m} \\
-\sum_{n=1}^{N} P Q_{p, n, t} ; \forall p, \forall t \\
I_{p, t}=I_{p, t-1}+\sum_{n=1}^{N} P Q_{p, n, t}-D_{p, t} ; \forall p, \forall t
\end{gathered}
$$

Mixing capacity constraint:

$$
\sum_{p=1}^{P} B_{p, m, t} \leq B P ; \forall m, \forall t
$$

Packing capacity constraint:

$$
\sum_{P \in S_{n}} P R_{p, n} \cdot P Q_{p, n, t} \leq A P_{n} ; \forall n, \forall t
$$

Max inventory level of intermediate product constraint:

$$
I M_{p, t} \leq I M \max _{p, t} ; \forall p, \forall t
$$

Safety stock constraint:

$$
I_{p, t} \geq \operatorname{Imin}_{p, t} ; \forall p, \forall t
$$

Cleaning constraint:

$$
\begin{gathered}
B_{p, m, t} \leq B P \cdot M M_{p, m, t} ; \forall p, \forall m, \forall t \\
P R_{p, n} \cdot P Q_{p, n, t} \leq A P_{n} \cdot M P_{p, n, t} ; \forall p, \forall n, \forall t
\end{gathered}
$$

The binary variables $\mathrm{MM}_{\mathrm{p}, \mathrm{m}, \mathrm{t}}$ and $\mathrm{MP}_{\mathrm{p}, \mathrm{n}, \mathrm{t}}$ in constraints 8 and 9 will be 1 if the mixing machine and packing machine are operated, respectively. When it is operated, it must be cleaned and the cleaning cost is included in the Eq. 1.

Non-negativity, binary, and integer conditions:

$$
B_{p, m, t}=\{0,1,2, \ldots\} ; \forall p, \forall m, \forall t
$$




$$
\begin{gathered}
P Q_{p, n, t}, I M_{p, t}, I_{p, t} \geq 0 ; \forall p, \forall n, \forall t \\
M M_{p, m, t}, M P_{p, n, t}=\{0,1\} ; \forall p, \forall m, \forall m, \forall t
\end{gathered}
$$

\subsection{Types of Planning Horizon Technique}

There are two types of planning horizons, namely, fixed and rolling horizons that are applied with the MILP model.

\subsubsection{Fixed Horizon Planning:}

The MILP model is solved once for the entire horizon of 30 daily periods.

\subsubsection{Rolling Horizon Planning:}

The entire horizon is divided into smaller sub-horizons as shown as an example in Fig. 2. In Fig. 2, there are 4 subhorizons with 10 periods each. Consecutive horizons should be overlapped for some periods to reduce the endof-horizon effect. Based on Fig. 2, the first sub-horizon covers periods 1 to 10 and the second one covers periods 8 to 17 . This means that periods 8 to 10 are overlapping periods. In this case the ending inventory of period 7 from the first sub-horizon will be used as the initial inventory of the second sub-horizon. The rolling horizon planning can still be divided into 2 types based on overlapping, namely, fixed and variable overlapping.

\subsubsection{The rolling horizon with fixed overlapping (RHFO)}

Uses the same length of overlapping periods for all consecutive sub-horizons. Fig. 2 shows the RHFO because the length of overlapping is three periods for all consecutive sub-horizons. RHFO which is applied in this paper is divided into 4 types based on the number of overlapping periods (1), which are 1, 2, 3, and 4 periods.

\subsubsection{The rolling horizon with variable overlapping ( $\mathrm{RHVO})$}

Allows different overlapping periods for each consecutive sub-horizon. Fig. 3 shows the RHVO because the lengths of overlapping are 3,2 , and 4 periods, respectively which are different. The RHVO is developed since the RHFO sometimes generates infeasible solutions. For example, when the overlapping period is set to 2 and the MILP model has infeasible solution, it is possible that if the overlapping period is changed to 1 or 3 , the MILP model may have a feasible solution.

There are many ways to vary overlapping periods. This paper set a systematic way to vary overlapping periods following "overlapping circulation numbers". For example, when the overlapping circulation numbers $(1,2,3,4)$ is used, the overlapping period of 1 will be tried first. If it has infeasible solution, the overlapping period will be 2 . If it is still infeasible, the next overlapping period will be tried until the last one. If all overlapping periods are tried but the solution is still infeasible, the solution is reported as infeasible.

There are 4 sets of the overlapping circulation numbers;

- $1,2,3,4$ circulation will be used if RHFO (l=1) generates infeasible solution.

- 2,3,4,1 circulation will be used if RHFO (l=2) generates infeasible solution.

- $3,4,1,2$ circulation will be used if RHFO (l=3) generates infeasible solution.

- 4,1,2,3 circulation will be used if RHFO (l=4) generates infeasible solution.

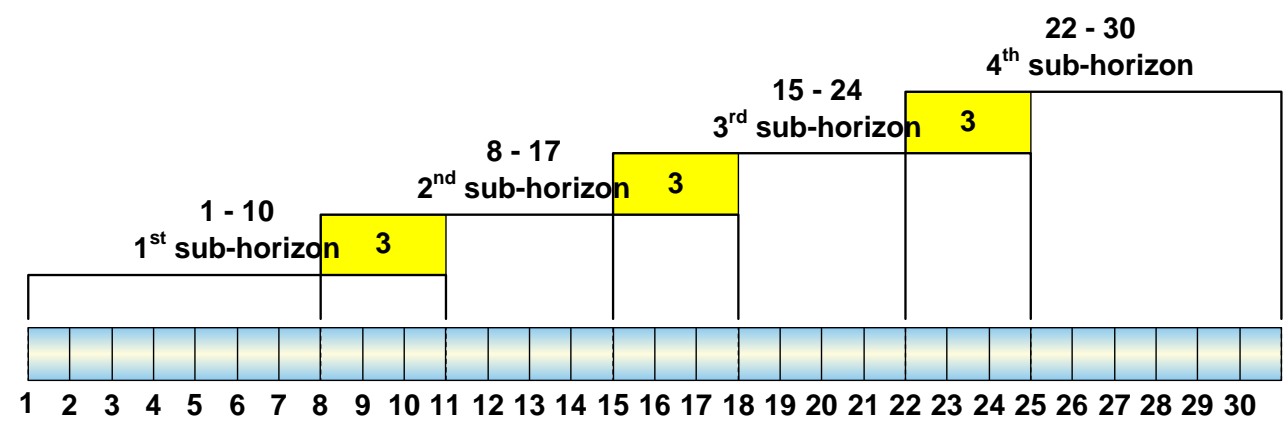

Fig. 2. Rolling horizon with fixed overlapping (RHFO) for 30 days in normal demand situation with 10 periods of each sub-horizon

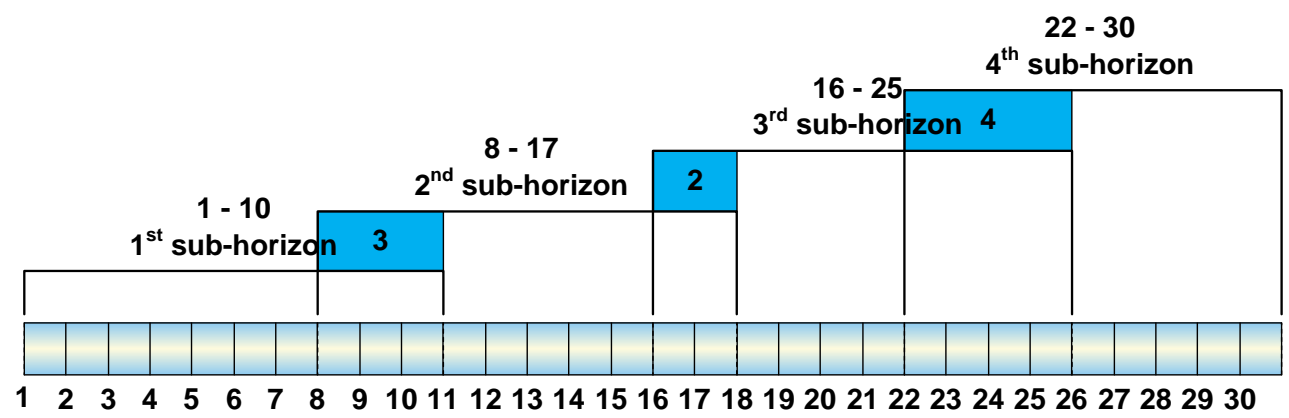

Fig. 3. Rolling horizon with variable overlapping (RHVO) for 30 days in normal demand situation with 10 periods of each sub-horizon 


\subsection{Experiments to Test Performances of Fixed and Rolling Horizon Planning Techniques}

There are two experiments to test performances of the fixed and rolling horizon planning techniques.

\subsubsection{Experiment 1: Performance of fixed horizon planning technique}

In this experiment, the MILP model will be solved using a planning horizon of 30 daily periods under low, normal, and high demand situations. This model requires very long computational time. Thus, it is solved for 6 hours and the solution quality is reported.

\subsubsection{Experiment 2: Performance of rolling horizon planning technique}

When the fixed horizon planning technique offers unsatisfactory solution quality for some demand situations, the MILP is solved using rolling horizon planning techniques. Then, the performances of both techniques are compared.

Detailed steps of both experiments are summarized in a flow chart in Fig. 4.

\section{Case Study}

This case study is performed in an adhesive plant. This adhesive plant is operated for 8 hours per day (8a.m.12p.m. and 1p.m.-5p.m.) and 22 days per month or 261 days per year. It has 4 adhesive products (A, B, C and D), which have different product formula. Therefore, the mixing machine must be cleaned when switching from producing one product to others to prevent contamination of different chemicals. The mixing time is still 3 hours per batch, although product formula of each adhesive product is different. All 4 products have similar pack size of about $350 \mathrm{ml}$. per tube.

A worker is used to clean mixing machine for 1 hour and material lost is about $0.5 \mathrm{~kg}$. The maximum number of mixing batches is 2 batches per day per machine or 4 batches per day for both machines. It has a normal practice to use a planning horizon of 30 working days. Fig. 1 shows the production process of adhesive. From the past data, the customer demands are classified to three (high, normal, and low) demand situations.

\subsection{Production Machines}

\subsubsection{Make stage}

There are a small set of mixing and pressing machines and a large set of mixing and pressing machines. The small set can produce 857 tubes per batch and the large set can produce 2,000 tubes per batch while the mixing and pressing times of both sets are the same which is 3 hours per batch.

\subsubsection{Intermediate stage}

The drums with a capacity of 200L are used to temporarily store the adhesive and wait for next packing process. There is unlimited numbers of available drums since it is inexpensive. The adhesive product can be stored in a drum for a long time because drums are completely sealed.

\subsubsection{Pack stage}

There are two packing machines. Packing rate of each machine is 10 tubes per minute or 4,800 tubes per day.
The packing machine number 1 is used for packing products $\mathrm{A}$ and $\mathrm{B}$. The packing machine number 2 is used for packing products $\mathrm{C}$ and $\mathrm{D}$.

\subsection{Estimation of Related Costs}

\subsubsection{Cleaning cost}

Material cost: The material will be lost during cleaning about $0.5 \mathrm{~kg}$ which is equivalent to $100 \mathrm{Baht}$ per time of cleaning.

Labor cost: The cleaning requires a worker, the cleaning time is about $1 \mathrm{hr}$, and the average labor cost is 37.50 Bath per man-hour, so the labor cost for cleaning is 37.50 baht.

Thus, the total cleaning cost is 137.5 baht/time.

\subsubsection{Production cost (exclude material cost)}

Small batch production cost: 10 baht per unit $(8,570$ baht per batch).

Large batch production cost: 7 baht per unit $(14,000$ baht per batch).

Note: Small batch cost is more expensive than large batch cost because of economy of scale.

\subsubsection{Holding cost}

Inventory holding cost of finished goods: It is equal to $30 \%$ of product value per year and product unit cost is 50 baht per tube (working days per year are 261).

holding cost per tube $=\frac{0.3}{\text { year }} \times \frac{50 \text { baht }}{\text { unit }}$

$$
\begin{aligned}
& =\frac{15 \text { baht }}{\text { year }(261 \text { days }) \times \text { unit }} \\
& =0.0575 \text { baht } / \text { unit } \cdot \text { day }
\end{aligned}
$$

Inventory holding cost of intermediate product: It is equal to $70 \%$ of holding cost of finish goods

$$
=0.04025 \mathrm{baht} / \mathrm{unit} \cdot \mathrm{day}
$$

\subsection{Values of Input Parameters}

Values of input parameters to the MILP model are summarized as follows.

$\boldsymbol{B} \boldsymbol{S}_{p, \mathbf{1}}$ batch size of mixing machine 1 for all products are 2,000 units

$\boldsymbol{B} \boldsymbol{S}_{\boldsymbol{p}, 2}$ batch size of mixing machine 2 for all products are 857 units

$\boldsymbol{C} \boldsymbol{P}_{\boldsymbol{p}, \mathbf{1}}$ production cost of mixing machine 1 for all products are 14,000 baht/batch

$\boldsymbol{C} \boldsymbol{P}_{\boldsymbol{p}, 2}$ production cost of mixing machine 2 for all products are $8,570 \mathrm{baht} / \mathrm{batch}$

$\boldsymbol{H M}_{\boldsymbol{p}}$ inventory holding cost of intermediate product for all products are $0.04025 \mathrm{baht} / \mathrm{unit}$-period

$\boldsymbol{H} \boldsymbol{F}_{\boldsymbol{p}}$ inventory holding cost of finished goods for all products are $0.0575 \mathrm{baht} / \mathrm{unit}$-period

$\boldsymbol{C C 1} \mathbf{1}_{\boldsymbol{p}}$ cleaning cost of mixing machines for all products is 137.5 baht

$C C 2_{p}$ cleaning cost of packing machines for all products is 137.5 baht 
$I M_{p, 0}$ initial inventory of intermediate product of product $\mathrm{p}$ (units) under normal demand situation

$$
\begin{array}{ll}
\mathrm{IM}_{1,0}=5,000 \text { units, } & \mathrm{IM}_{2,0}=5,400 \text { units, } \\
\mathrm{IM}_{3,0}=8,200 \text { units, } & \mathrm{IM}_{4,0}=5,100 \text { units }
\end{array}
$$

$I_{\max }, t$ maximum inventory level of intermediate product of product $\mathrm{p}$ in period $\mathrm{t}$ (units) under normal demand situation

$\operatorname{IMmax}_{1, \mathrm{t}}=5,027$ units for all $\mathrm{t}$,

$\operatorname{IMmax}_{2, \mathrm{t}}=5,459$ units for all $\mathrm{t}$,

$\operatorname{IMmax}_{3, \mathrm{t}}=8,224$ units for all $\mathrm{t}$,

$\operatorname{IMmax}_{4, \mathrm{t}}=5,138$ units for all $\mathrm{t}$

$I_{p, 0} \quad$ initial inventory of product $\mathrm{p}$ (units) under normal demand situation

$\mathrm{I}_{1,0}=19,900$ units,

$\mathrm{I}_{2,0}=18,900$ units,

$\mathrm{I}_{3,0}=23,900$ units,

$\mathrm{I}_{4,0}=24,900$ units

$\operatorname{Imin}_{p, t}$ minimum inventory level of product $\mathrm{p}$ in period $\mathrm{t}$ (units) under normal demand situation

$\operatorname{Imin}_{1, \mathrm{t}}=19,230$ units for all $\mathrm{t}$,

$\operatorname{Imin}_{2, \mathrm{t}}=18,779$ units for all $\mathrm{t}$,

$\operatorname{Imin}_{3, \mathrm{t}}=23,445$ units for all $\mathrm{t}$,

$\operatorname{Imin}_{4, \mathrm{t}}=24,675$ units for all $\mathrm{t}$

$\boldsymbol{B P}$ maximum number of mixing batches per period is 2 batches/period-machine

$\boldsymbol{P} \boldsymbol{R}_{\boldsymbol{p}, \boldsymbol{n}}$ unit packing time for all products on all packing machines is 0.1 minute/unit

$\boldsymbol{A P}_{\boldsymbol{n}} \quad$ available packing time for all packing machines is 480 minutes/day

$\boldsymbol{S}_{\boldsymbol{n}} \quad$ set of products that can be packed on packing machine $\mathrm{n}$

$\mathrm{S}_{1}=\{\mathrm{A}, \mathrm{B}\}, \quad \mathrm{S}_{2}=\{\mathrm{C}, \mathrm{D}\}$

$\boldsymbol{D}_{\boldsymbol{p}, \boldsymbol{t}}$ demand of product $\mathrm{p}$ in period $\mathrm{t}$ under normal demand situation (units) is shown in Table 1. Since there is a page limit, the demands in low and high demand situations are not shown.

\section{Results and Discussion}

\subsection{Experiment 1: Performance of Fixed Horizon Planning Technique}

After The MILP is solved for 6 hours, the solutions for each demand situations are reported in Table 2. The optimal solutions are still not obtained. The total costs are reported and compared with their lower bounds. During high demand situation, the gap of $1.886 \%$ is satisfactory. However, during low and normal demand situations, the gaps are too high and the solution qualities are unsatisfactory. Thus, the rolling horizon planning technique will be used for the low and normal demand situations.

\subsection{Experiment 2: Performance of Rolling Horizon Planning Technique}

Table 3 shows performances of rolling horizon planning for low demand situation. In each cell of table 3 , the first line shows the lengths of sub-horizons and overlapping periods in the first and second parentheses, respectively. For example, $(10,10,10,4)(1,1,2)$ means that the lengths of the first to fourth sub-horizons are 10, 10, 10, and 4 periods, respectively, and the overlapping periods between consecutive sub-horizons are 1,1 , and 2 periods. The second line shows total computational time and the last line shows total cost with the gap between the total cost and the lower bound of the total cost obtained from the fixed horizon planning method in parenthesis.

Table 3 shows that there are 5 out of 12 solutions that the rolling horizon planning method has lower total cost than the fixed horizon planning method. The best total cost has the gap of $4.62 \%$ which is slightly better than the fixed horizon one that has a gap of $5.136 \%$. There is a case that the RHFO cannot generate a feasible solution but the RHVO can generate the feasible solution. However, the solution of RHVO is worse than the solution from the fixed horizon planning method. The rolling horizon planning method can significantly save computational time when compared with the fixed horizon planning method.

Performances of rolling horizon planning method for normal demand situation are presented in Table 4 . Table 4 shows that there are 11 out of 12 solutions that the rolling horizon planning method has lower total cost than the fixed horizon planning method. There is a case that the rolling horizon planning method has an infeasible solution. The best total cost has the gap of $3.152 \%$ which is greatly better than the fixed horizon one that has a gap of $7.8 \%$. There are three cases that the RHFO cannot generate a feasible solution but the RHVO can generate the feasible solutions which are better than those from the fixed horizon planning method. The rolling horizon planning method can significantly save computational time when compared with the fixed horizon planning method.

Based on the experimental results, when the fixed horizon planning method gets the solution with relatively large gap between the total cost and the lower bound of the total cost, the quality of solution can be improved by using the rolling horizon planning method. Two parameters (length of sub-horizon and overlapping periods) of the rolling horizon planning method affect the quality of solution and total computational time. Relatively long sub-horizon (15 and 17 periods) tends to have better solution quality than relatively short sub-horizon (10 periods). It also has shorter total computational time. Relatively long overlapping periods tends to have better solution quality. Although RHVO can generate feasible solution in some cases that RHFO results in infeasible solution, RHVO cannot generate the best solution.

\section{Conclusions}

The MILP model is specially developed to fit requirements of make-pack production of adhesive factory. The solution obtained from the model is reasonable and useful for production planning and scheduling. However, the entire planning horizon of 30 daily periods results in many binary variables in the model. This requires very long computational time. The solution obtained within reasonable computational time (6 hours) has relatively large gap from the lower bound for some demand situations. In this case, the rolling horizon planning method can be used to improve the quality of solution and reduce computational time. The length of sub-horizon and 
overlapping periods affect the quality of solution and computational time. The rolling horizon planning method is also suitable for dynamic situations that customer demands are changed frequently or the production plan is needed to be changed often.

There are some interesting issues that need to be studied in the future. Firstly, the MILP model may be extended to handle uncertain or fuzzy parameters. Some fuzzy techniques may be used to improve the performances of scheduling methods. Kabir and Sumi (2013) demonstrated that fuzzy techniques, namely fuzzy Delphi and fuzzy AHP methods can be used to enhance performances of inventory classification process. Secondly, the performances of rolling horizon planning method should be tested using many data sets to verify whether it will consistently outperform the fixed horizon planning method.
Note :

$I=$ The number of periods is overlapped between two sub-horizons

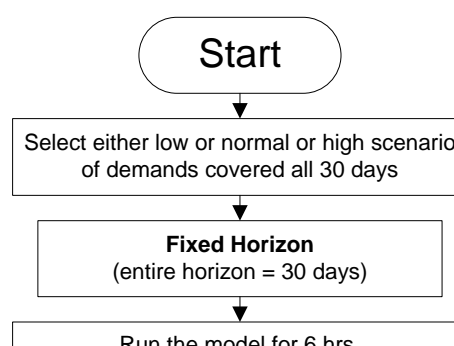

Run the model for $6 \mathrm{hrs}$ And \% different of current best solutions from lower bound are recorded every 30 minutes

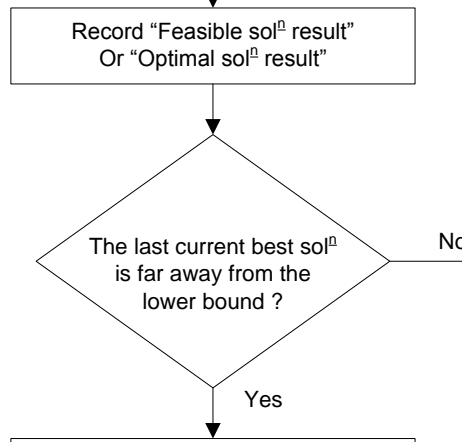

Select sub-horizon size equal either to 10 or 15 or 17 days to be rolled horizon

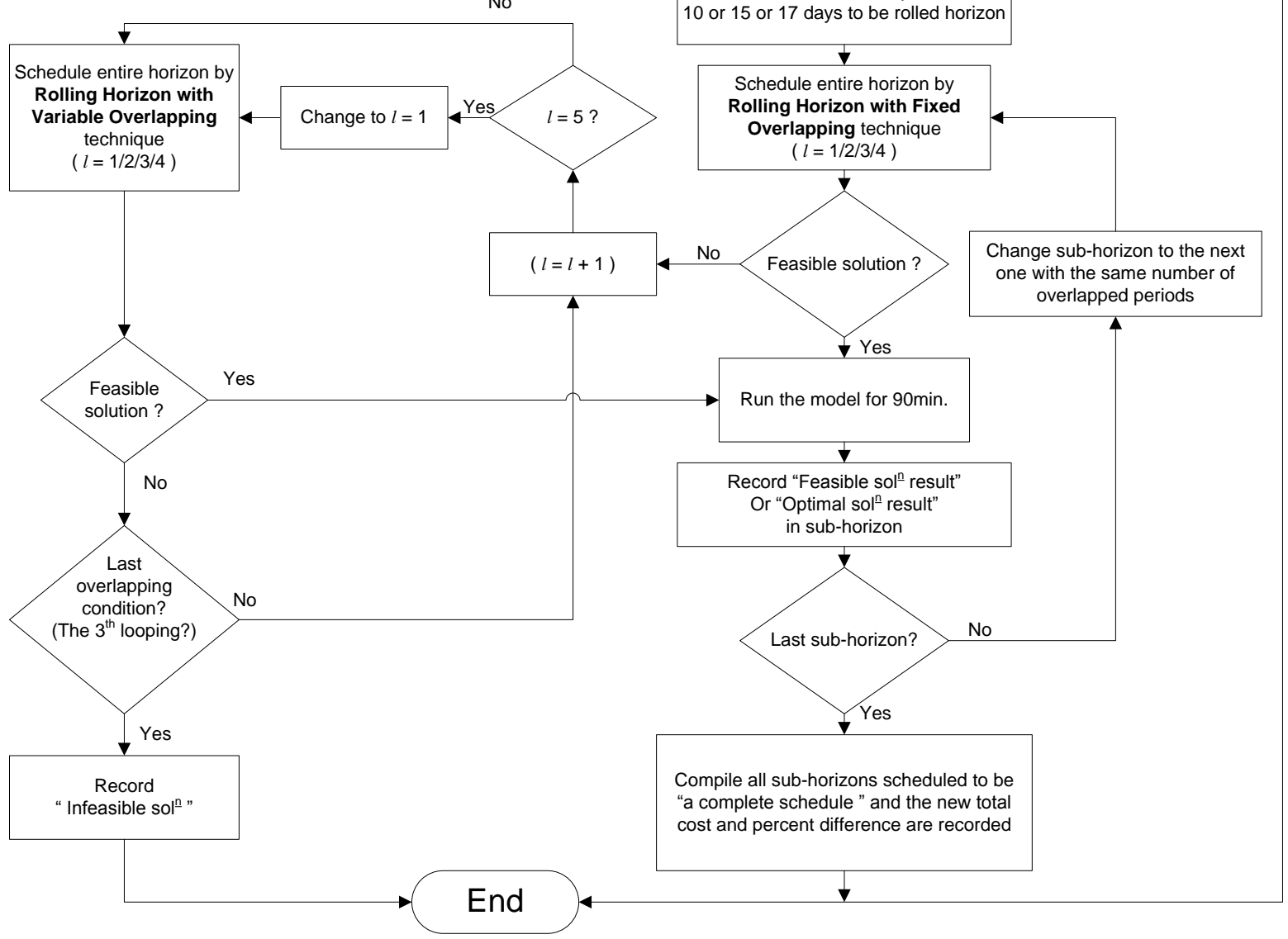

Fig. 4. Flow chart of experiment 
Table 1. Normal demand situation of each product in each period

\begin{tabular}{|c|c|c|c|c|}
\hline Period & Product A & Product B & Product C & Product D \\
\hline 1 & 0 & 0 & 0 & 0 \\
\hline 2 & 0 & 0 & 2311 & 0 \\
\hline 3 & 767 & 516 & 2600 & 976 \\
\hline 4 & 0 & 1560 & 5460 & 0 \\
\hline 5 & 0 & 0 & 0 & 0 \\
\hline 6 & 6481 & 5897 & 2600 & 750 \\
\hline 7 & 0 & 2340 & 0 & 2340 \\
\hline 8 & 0 & 0 & 0 & 0 \\
\hline 9 & 0 & 0 & 7540 & 0 \\
\hline 10 & 1227 & 0 & 0 & 0 \\
\hline 11 & 2717 & 1950 & 1690 & 8892 \\
\hline 12 & 0 & 2340 & 0 & 2340 \\
\hline 13 & 0 & 0 & 0 & 0 \\
\hline 14 & 1300 & 517 & 2600 & 750 \\
\hline 15 & 0 & 516 & 2600 & 534 \\
\hline 16 & 0 & 0 & 7540 & 0 \\
\hline 17 & 1300 & 517 & 2600 & 750 \\
\hline 18 & 0 & 0 & 0 & 0 \\
\hline 19 & 5181 & 5379 & 0 & 0 \\
\hline 20 & 0 & 0 & 2311 & 0 \\
\hline 21 & 767 & 0 & 0 & 442 \\
\hline 22 & 0 & 516 & 2600 & 543 \\
\hline 23 & 0 & 0 & 0 & 0 \\
\hline 24 & 2717 & 1950 & 1690 & 8892 \\
\hline 25 & 0 & 516 & 2600 & 543 \\
\hline 26 & 5181 & 5379 & 0 & 0 \\
\hline 27 & 0 & 2340 & 0 & 2340 \\
\hline 28 & 0 & 0 & 0 & 0 \\
\hline 29 & 1300 & 517 & 2600 & 750 \\
\hline 30 & 1227 & 0 & 0 & 0 \\
\hline
\end{tabular}

Table 2. Solution quality of fixed horizon planning for three customer demand situations

\begin{tabular}{cccc}
\hline Demand situations & Low & Normal & High \\
\hline Production cost (B) & 694,570 & 909,410 & $1,091,670$ \\
Inventory cost (B) & 127,044 & 171,562 & 155,854 \\
Cleaning cost (B) & $10,312.5$ & $12,237.5$ & 13,750 \\
Total cost (B) & $831,926.5$ & $1,093,209.5$ & $1,237,274$ \\
Lower bound of total cost (B) & $791,283.0$ & $1,014,110.0$ & $1,237,920$ \\
Gap between total cost and lover bound (\%) & 5.136 & 7.800 & 1.886 \\
\hline
\end{tabular}


Table 3. Solution quality of rolling horizon scheduling in low demand situation

\begin{tabular}{cccc}
\hline \multirow{2}{*}{$\begin{array}{c}\text { Overlapping } \\
\text { circulation number }\end{array}$} & \multicolumn{3}{c}{ Sub-horizon length (periods) } \\
\cline { 2 - 4 } & 10 & 15 & 17 \\
\hline $1,2,3,4$ & $(10,10,10,4)(1,1,2) \S$ & $(15,15,2)(1,1)$ & $(3 \mathrm{hr})$ \\
& $(4.5 \mathrm{hr})$ & $(3 \mathrm{hr})$ & $834,031(5.402 \%)$ \\
$2,3,4,1$ & $843,271.5(6.570 \%)$ & $830,593.7(4.968 \%) *$ & $(17,15)(2)$ \\
& $(10,10,10,6)(2,2,2)$ & $(15,15,4)(2,2)$ & $(3 \mathrm{hr})$ \\
$3,4,1,2$ & $(4.5 \mathrm{hr})$ & $(3 \mathrm{hr})$ & $832,150(5.165 \%)$ \\
& $839,587.8(6.105 \%)$ & $829,810.4(4.869 \%) *$ & $(17,16)(3)$ \\
& $(10,10,10,9)(3,3,3)$ & $(15,15,6)(3,3)$ & $(3 \mathrm{hr})$ \\
& $(6 \mathrm{hr})$ & $(3 \mathrm{hr})$ & $831,819(5.123 \%)$ \\
& $837,109(5.791 \%)$ & $830,656.6(4.976 \%) *$ & $(17,17)(4)$ \\
& $(10,10,10,10),(4,4,4,4)$ & $(15,15,8)(4,4)$ & $(3 \mathrm{hr})$ \\
\end{tabular}

* means the total cost is less than the fixed horizon planning method

** means the lowest total cost

$\S$ RHVO is used

Table 4. Solution quality of rolling horizon scheduling in normal demand situation

\begin{tabular}{|c|c|c|c|}
\hline \multirow{2}{*}{$\begin{array}{c}\text { Overlapping } \\
\text { circulation number }\end{array}$} & \multicolumn{3}{|c|}{ Sub-horizon length (periods) } \\
\hline & 10 & 15 & 17 \\
\hline \multirow[t]{3}{*}{$1,2,3,4$} & $(10,10,10,6)(3,1,2) \S$ & $(15,15,3)(2,1) \S$ & $(17,14)(1)$ \\
\hline & $(4.5 \mathrm{hr})$ & $(3 \mathrm{hr})$ & $(3 \mathrm{hr})$ \\
\hline & $1,077,221(6.223 \%) *$ & $1,069,537(5.466 \%) *$ & $1,046,529(3.197 \%) *$ \\
\hline \multirow[t]{3}{*}{$2,3,4,1$} & $(10,10,10,9)(3,2,4) \S$ & $(15,15,4)(2,2)$ & $(17,15)(2)$ \\
\hline & $(6 \mathrm{hr})$ & $(3 \mathrm{hr})$ & $(3 \mathrm{hr})$ \\
\hline & $1,077,386(6.240 \%) *$ & $1,069,375(5.450 \%) *$ & $1,046,753(3.219 \%) *$ \\
\hline \multirow[t]{3}{*}{$3,4,1,2$} & $(10,10,10,9)(3,3,3)$ & $(15,15,6)(3,3)$ & $(17,16)(3)$ \\
\hline & $(6 \mathrm{hr})$ & $(3 \mathrm{hr})$ & Infeasible \\
\hline & $1,077,302(6.231 \%) *$ & $1,058,193(4.347 \%) *$ & \\
\hline \multirow[t]{3}{*}{$4,1,2,3$} & $(10,10,10,10,6)(4,4,4,4)$ & $(15,15,8)(4,4)$ & $(17,17)(4)$ \\
\hline & $(6 \mathrm{hr})$ & $(4.5 \mathrm{hr})$ & $(3 \mathrm{hr})$ \\
\hline & $1,073,743(5.880 \%) *$ & $1,046,070(3.152 \%) * *$ & $1,047,355(3.278 \%) *$ \\
\hline
\end{tabular}

* means the total cost is less than the fixed horizon planning method

** means the lowest total cost

$\S$ RHVO is used

\section{References}

Enns, S. T. V. (1996). Finite capacity scheduling systems: performance issues and comparisons, Computers and industrial engineering, 30, 727-739.

Fang, J. and Xi, Y. (1997). A rolling horizon job shop rescheduling strategy in the dynamic Environment, The International Journal of Advanced Manufacturing Technology, 13, 227-232.

Fündeling, C.-U. and Trautmann, N. (2006). Scheduling of make and pack plants: a case study. Computer Aided Chemical Engineering, 21, 1551-1556.

Günther, H.-O., Grunow, M., and Neuhaus, U. (2006). Realizing block planning concepts in make-and-pack production using MILP modeling and SAP APO,
International journal of Production Research, 44, 3711-3726.

Honkomp, S. J., Lombardo, S., Rosen, O., and Pekny, J. F. (2000). The curse of reality - why process scheduling optimization problems are difficult in practice, Computers and Chemical Engineering, 24, 323-328.

Kabir, G. and Sumi, R. S. (2013). Integrating fuzzy Delphi with fuzzy analytic hierarchy process for multiple criteria inventory classification, Journal of Engineering, Project, and Production Management, 3(1), 22-34.

Kreipl, S. and Pinedo, M. (2004). Planning and Scheduling in Supply Chains: An Overview of Issues 
in Practice, Production and Operations Management Society, 13, 77-92.

Liu, S. and Pinto, J. M. (2010). MILP-based approaches for medium-term planning of single-stage continuous multiproduct plants with parallel units, Computational Management Science, 7, 407-435.

Méndez, C. A. and Cerdá, J. (2002). An MILP-based approach to the short-term scheduling of make-andpack continuous production plants, OR Spectrum, 24, 403-429.

Nagendra, P. B. and Das, S. K. (2001). Finite capacity scheduling method for MRP with lot size restrictions, International Journal of Production Research, 39, 1603-1623.

Stauffer, L. and Liebling, Th. M. (1997). Rolling horizon scheduling in a rolling-mill, Annals of Operations Research, 69, 323-349.

Sun, H. L. and Xue, Y. F. (2009). An MILP formulation for optimal scheduling of multi-product batch plant with a heuristic approach, The International Journal of Advanced Manufacturing Technology, 43, 779-784.

Wongthatsanekorn, W., Phruksaphanrat, B., and Sangkhasuk, R. (2013). Applying Bee Colony Optimization Heuristic for Make-Pack Problem in Process Manufacturing, KKU Engineering Journal, 40(1), 47-56.

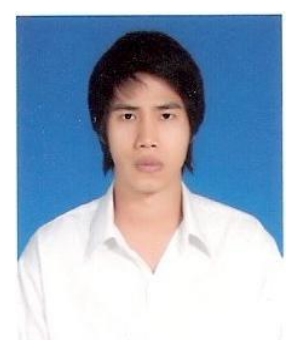

Mr. Theppakarn Chotpradit is a Master Student in Logistics and Supply Chain Systems Engineering Program at Sirindhorn International Institute of Technology (SIIT), Thammasat University. He holds B.Sc. in Food Science and Technology from Chiang Mai University. His research interests focus on production planning and inventory control systems, rolling horizon scheduling, scheduling optimization, and finite capacity scheduling systems.

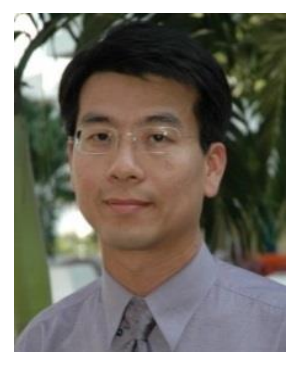

Dr. Pisal Yenradee is an Associate Professor in Industrial Engineering Program at Sirindhorn International Institute of Technology (SIIT), Thammasat University. He holds a B.Eng. in Production Engineering from King Mongkut's Institute of Technology and M.Eng. and D.Eng. in Industrial Engineering and Management from Asian Institute of Technology. His research interests focus on production planning and inventory control systems, JIT, ERP, TOC, finite capacity scheduling systems, aggregate production planning, master production scheduling, supply chain, and applied operations research. He has published papers in and served as a reviewer for many international journals. 